\title{
Nonketotic hyperglycemia-related epileptic seizures
}

\author{
Xuejian Wang
}

\begin{abstract}
Objective: To investigate nonketotic hyperglycemia (NKH)-related epileptic clinical features and pathogenesis, and improve the diagnosis and treatment.

Methods: Clinical data, including the clinical manifestations, laboratory tests, imaging studies and other information, of 13 patients with hyperglycemia-related epilepsy in our department were retrospectively analyzed.

Results: Blood glucose levels of the 13 patients when admitted to the hospital ranged between $24.7-34.6 \mathrm{mmol} / \mathrm{L}$ (average $28.3 \mathrm{mmol} / \mathrm{L}$ ), their plasma osmolality ranged between $290-332 \mathrm{mOsm} / \mathrm{L}$ (average $308 \mathrm{mOsm} / \mathrm{L}$ ), and their ketone results were negative. Among them, seven had convulsions, 4 had upper limbs and facial twitching, and 2 had bust twitch. Imaging findings could not detect accountable lesions related to seizures. EEG mainly showed spikes, slow waves, and scattered sharp slow waves. Insulin combined short-term antiepileptic drugs, allowed the epilepsy to be effectively controlled without recurrence.

Interpretation: Patients with episodes of NKH epilepsy increased significantly with hyperglycemia. Raising awareness of the disease, early diagnosis, and very early lowering the hyperglycemia levels, can effectively control the seizures. Lowering blood glucose is an effective way to control blood glucose levels.
\end{abstract}

Keywords: Nonketotic hyperglycemia, Seizure, Etiology, Epileptic seizures, Diabetic

Nonketotic hyperglycemia (NKH)-related epileptic seizures can be diagnosed when high blood glucose accompanied with normal plasma osmolality and negative urine ketone occurs. Its initial symptoms are seizures. They are clinically rare, and patients might not have prior history of diabetes or onset of diabetic symptoms. Because epileptic seizures related to NKH significantly affect neurological outcomes and may cause misdiagnosis or missed diagnosis, more attention should be paid to them. In this article, NKH epilepsy patients, who were treated in our department from December 2009 to October 2014, were clinical analyzed, in order to facilitate the future treatment.

\section{Material and methods}

\section{Ethics statement}

This research has been approved by the ethics committee of the Second Hospital affiliated to Nantong University.

\section{Correspondence: 6841441@163.com.cn}

Department of Neurosurgery, The second Hospital affiliated to Nantong

University, Nantong University, Jiangsu 226001, China
Informed consent has been obtained and this investigation has been conducted according to the principles expressed in the Declaration of Helsinki.

\section{General information}

Thirteen cases of NKH-related epileptic seizures were treated from December 2009 to October 2014 in our neurosurgery department. Among them, 8 were males, and 5 females, aged $45-80$ years old (mean age: $54 \pm$ 2 years old). Their diabetes duration was between 0 and 10 years (mean 4.7 years). Diagnosis of diabetes was performed according to the WHO diagnostic criteria. The results showed that 9 patients had type 2 diabetes, and 5 had a history of type 2 diabetes. None of them had history of contacting the toxic substances, epilepsy, or family history of epilepsy.

\section{Signs and symptoms}

Convulsive site during epilepticus insultus: convulsions occurred in seven cases, with 4 of them having upper limb and facial twitching, and 2 with bust twitch. 
Unconsciousness seizures occurred in eight cases, with the onset of the interval from $10 \mathrm{~min} \sim 2 \mathrm{~h}$, lasting for 4-20 min. Among those 8 cases, five were in persistent state.

\section{Supplementary examination}

Head imaging test did not find lesions that accounted for seizures. Laboratory tests at the admission: several cases with levels of venous blood glucose and peripheral blood glucose considerablely higher than the critical value of the for diabetes diagnosis, blood glucose 24.734.6 (average 28.3 ) $\mathrm{mmol} / \mathrm{L}$; plasma osmolality $296-322$ (average 308) $\mathrm{mOsm} / \mathrm{L}$, did not reach the diabetic hyperosmolar (>350 mOsm/L) of the diagnostic criteria. When seizures were under control, average blood glucose was $13.6 \mathrm{mmol} / \mathrm{L}$. Potassium, calcium and phosphorus were generally within the normal ranges, and blood urea nitrogen and serum creatinine also in the normal range. EEG showed spike, slow waves, scattered sharp slow wave in 8 cases.

\section{Treatment}

Once newly epileptic seizure was diagnosed, antiepileptic treatment was immediately conducted. Intramuscular administration of phenobarbital, diazepam and valproate, or valproate combined with carbamazepine was applied in defined cases, but with suboptimal results. After blood glucose levels increased, insulin infusion or subcutaneous injection were immediate applied. Close monitoring of blood glucose, saline rehydration and aggressiveness simultaneously corrected acid-base unbalance and water and electrolyte disorders. If blood glucose levels dropped to $8-15 \mathrm{mmol} / \mathrm{L}$ and epileptic episodes stopped, the antiepileptic drugs would be discontinued.

\section{Results}

The selected 13 patients were cured, as their seizures disappeared. The treatment procedures lasted 11-76 h (average $23 \mathrm{~h}$ ). After reduction of the blood glucose level by insulin, rehydration, and control of epilepsy, the number of seizures gradually reduced. The patients' condition gradually improved and eventually regained the consciousness without nervous system dysfunction left. The average blood glucose when epileptic seizures ended was $10 \mathrm{mmol} / \mathrm{L}$. Anti-epileptic drugs were not prescribed after discharge. During the two-month to oneyear follow-up, no recurrent seizure appeared.

\section{Discussions}

Focal seizures (also known as partial seizure) induced by hyperglycemia were first reported by Maccario et al in 1965 [1-3]. They were often encountered in clinical practice and characterized by hyperglycemia without keto-acidosis. Seizure control associated with resolution of the hyperglycemia was usually used to manage the partial seizures [2, 4]. Many studies have described this clinical syndrome [5-7]. In recent years, clinical reports gradually increased about NKH-related epileptic seizures, which are considered one of the major neurological complications of diabetes [3, 8]. It indicates an unsatisfactory blood glucose control if $\mathrm{NKH}$-related epileptic seizures occur [6].

For patients with seizures as main manifestations, especially those without a history of diabetes, the initial diagnosis would often be neurology-related. If conventional antiepileptic treatment is applied, the results would be more harmful than beneficial, and in few ill cases, increased mortality might be observed. The possible explanations include: (1) these patients are more frequently accompanied with unconsciousness and seizures. The preferred drugs, such as diazepam and phenobarbital that ends epileptic episode, often aggravated the unconscious patients by causing respiration inhibition, making the disease more complicated and affecting the subsequent diagnosis and treatment; (2) treatment of intractable seizures uses anti-epilepsy medicine that involves multi-combination of mannitol, corticosteroids and other drugs to reduce brain edema; however, the risk factors of the focal seizure disease are high-blood glucose, high permeability and intracellular dehydration. These drugs will inevitably aggravate the patient's condition; (3) NKH: featured with reduction of brain $\gamma$-aminobutyric acid content, and therefore diazepam and phenobarbital actually decreased the antiepileptic effect; (4) Since glucose saline injection is commonly used as intravenous drugs in the absence of insulin, it might worsen the condition. The disease tend to cause seizures in some patients without diabetes history, and therefore it is more difficult to catch the attention, and more likely to be misdiagnosed.

Diabetes is the most common cause of the seizures in patients with low blood glucose. The subsequent unconsciousness with ketosis acidosis and NKH coma are more common in clinical practice; however, high blood glucose can also lead to seizures, even status epilepticus without awareness. Careful analysis the patients who had epilepsy, accompanied with occasional onset of unconsciousness in this group, first found significantly increased biochemical blood glucose levels, leading to the exclusion of the possibility of hypoglycemia. Diabetes can be complicated by acidosis or ketosis ketotic hyperosmolar coma. As their urine ketone was negative, the possibility of ketosis acidosis could next be ruled out. Blood biochemistry showed that their glucose, sodium, and potassium, were only slightly elevated, and so was the blood urea nitrogen content in plasma osmolality values inferred, which was less than $350 \mathrm{mOsm} / \mathrm{L}$, nonketotic diabetic hyperosmolar coma was also excluded. 
Therefore, NKH-related epileptic seizures should be next considered. The seizures pathogenesis caused by this disease is still controversial, as some people think that the main pathogenesis is the lack of insulin. Among the patients, insulin levels are sufficient to inhibit the free fatty acid metabolism and the subsequent ketoacidosis, but not enough to transport glucose into the cells [9]. High blood glucose levels increase the levels of urine glucose, causing osmotic diuresis effect and progressive dehydration, which would in turn increase the incidence of this disease. Some people believe that the possible mechanisms include high blood glucose, high plasma osmolality and $\gamma$ - aminobutyric acid (GABA) levels, and low focal cerebral ischemia [8]. Specific mechanisms also include: (1) osmotic changes. Hyperglycemia causes significant and rapid increase of intracellular osmotic pressure, leading to nerve cell dehydration, and changes in enzyme activity and brain cell energy metabolism. Membrane ion pump function is impaired, causing the loss of intracellular potassium and the subsequent sodium accumulation, which destroys the membrane potential and the stability of cell depolarization, ultimately resulting in seizures [4]. (2) in vivo biochemical changes in metabolism. Citric acid cycle is inhibited in vivo in the patients with this disease, whereas GABA metabolism is increased, causing the increased brain energy consumption, and the reduced seizure threshold [10]. In contrast, seizures are less frequent in ketoacidosis patients, as ketosis acidosis increases intracellular activities of glutamate and tryptophan decarboxylase, leading to the increased content of brain inhibitory neurotransmitter GABA. GABA is related to the rapid changes of synaptic sensitivities by binding to the neurons, which then increases the permeability of chloride ions. Under such conditions, the membrane potential is maintained at a stable resting potential level and the excitatory synaptic reactivities are weakened so that the epilepsy is prevented. This is also an alternative way to prove that the GABA contents decrease in NKH-related epileptic seizures patients. (3) brain cell energy deficiency. Because of the diabetic hyperglycemia, plasma fibrinogen significantly increases, red blood cell and platelet aggregate, and blood is in a hypercoagulable state. In addition, the aggravation of existing diabetes microcirculation and small artery hyalinization, the dysfunction of endothelial cells, and damages of cerebral blood flow autoregulation, decrease regional cerebral blood flow, causing hypoxicischemic damages and functional changes in cortical cells, which are "epilepsy cells". Such cells are sensitive to the metabolic disorders, high blood glucose condition especially is likely to cause seizures (4) immune abnormalities: the presence of glutamic acid decarboxylase autoantibodies in both type 1 diabetes and epilepsy. Peltola, et al studied 51 cases of refractory epilepsy and found that these patients were autoantibody positive, with antibody titers similar to that in patients with type 1 diabetes, suggesting that intrinsic link might exist between them Their results supported the idea that immune dysfunction was involved in the hyperglycemiarelated epilepsy [11] (5) studies suggest that NKHrelated seizures might be linked to the brain barrier damage caused by long-term high blood glucose [12]. However, most of patients do not have seizures even if their blood glucose levels are high as the high levels of blood glucose are not the only factor for epilepsy. Other factors include individual health status and genetic factors [7]. In 1968, Maccario et al. [2, 3] first reported that $\mathrm{NKH}$ and partial seizures co-existed, which were featured with high blood glucose, absence of ketosis, consciousness, and partial seizures. Tiamkao et al. [8], based on a retrospective analysis of 21 partial NKHrelated seizures patients, found that the average blood glucose level was $32.6 \mathrm{l} \mathrm{mmol} / \mathrm{L}(16.11 \sim 61.33 \mathrm{mmol} / \mathrm{L})$, and mean plasma osmolality was $302 \mathrm{mOsm} / \mathrm{L}$ (288$323 \mathrm{mOsm} / \mathrm{L}$ ). When the seizure was under control, the average of blood sugar level was $11.3 \mathrm{mmol} / \mathrm{L}$ (4.11$21.67 \mathrm{mmol} / \mathrm{L})$.

This paper reported eight cases with EEG showing spikes, slow waves, and scattered sharp slow waves. In previously published reports, EEG results of patients with $\mathrm{NKH}$-related epileptic seizures have shown normal waves or spikes, sharp slow or high amplitude slow waves [13]. Therefore, EEG is not very valuable in diagnosis of the disease. Imaging is not specific, either. This group mostly showed the normal imaging results, or age-related abnormalities such as old lacunar infarction, brain atrophy, and white matter demyelination, similar to most of the previous studies [5, 6]. However, some studies [14-16] described a transient change in head MRI in a patient with $\mathrm{NKH}$-induced seizures, that is, the reversible and Flair weighted hyperintense cortex, and low signal of white matter. He also displayed cytotoxic edema. Its mechanism was unclear. Possibly, the cortical ischemia seizures and angioedema, caused aggregation and deposition of iron radicals [14-16]. Some scholars believe that these changes result from the damages in blood-brain barrier caused by long-term high levels of blood sugar [12].

This article summarized the following clinical features of NKH-related epileptic seizures: (1) common in the elderly (2) with or without a previous history of diabetes and epilepsy (3). Seizures were always accompanied with a rapid rise in blood glucose. Plasma osmolality may be normal or slightly elevated, but not to the diagnostic criteria for diabetes hypertonic (4) urine ketone negative (5). Seizures could not be effectively alleviated by antiepileptic drugs alone. Application of insulin to correct hyperglycemia and metabolic disorders ended the seizures (6) 
seizure-related lesions were not detected in the head imaging tests (7). If blood glucose was under control, epilepsy did not occur any more. Analysis from the clinical effectiveness showed that the primary treatment for the $\mathrm{NKH}$-related epileptic seizures included early, active, and rational rehydration and insulin hypoglycemic therapy, while closely monitoring blood glucose. Such treatments are key to the success of salvage therapy among these patients. Phenytoin-induced insulin resistance can inhibit the release of insulin, and therefore increase the possibility of NKH-related seizures. Diazepam increase the opening frequency of GABA-mediated chloride ion channel. Phenobarbital extended the start time of GABA-mediated chloride channel, by reducing the brain GABA levels among the patients with NKH-related seizures, Therefore, the stability and antiepileptic effects of phenobarbital and Diazepam decline $[2,5-7]$ which explained why conventional antiepileptic drugs in those patients with epilepsy were not marketly effective. Antiepileptic drugs preferred include carbamazepine, clonazepam diazepam and other anti-epileptic drugs as they do not affect the levels of blood glucose. Long-term use of anti-epileptic drugs is not necessary. If blood glucose and the seizures are well controlled, anti-epileptic drugs can be discontinued gradually. Smooth phasing out the anti-epileptic drugs among the patients in the present study did not cause the recurrence.

In short, NKH-related epileptic seizures has low occurrence rate in clinical practice. Epilepsy is often the first symptom. Compared with other diseases presenting clinical manifestations of epilepsy, they are not specific, and tend to be misdiagnosed. Therefore, early recognition of this clinical syndrome and other seizure causes as well as early phase identification is critical, because the condition can be corrected by adjusting blood glucose level plus rapid rehydration. The disease pathogenesis is not entirely clear. In order to clarify the clinical features and pathogenesis of this disease, further research should be carried out in the imaging and electrophysiological aspects.

\section{Acknowledgements}

We didn't receive any financial support to analyze this data and we didn't have any financial interest in any materials or devices described.

\section{Funding}

This research supported by: (1) Young Medical Project of Nantong City Health Bureau(WQ2014016); (2) Traditional Chinese medicine science and technology project in Jiangsu province(No. YB2015113); (3) the science and technology program of Nantong city (no. : MS12015016).

Availability of data and materials

Please contact author for data requests.

Author's contributions

All by Xuejian Wang.

Competing interests

The author declares that he has no competing interests.

\section{Consent for publication}

All authors have approved the manuscript and agree with submission to Journal of Chinese Neurosurgical Journal.

Ethics approval and consent to participate

This research has been approved by the ethics committee of the Second Hospital affiliated to Nantong University. Informed consent has been obtained and this investigation has been conducted according to the principles expressed in the Declaration of Helsinki.

Received: 8 September 2016 Accepted: 10 January 2017

Published online: 03 April 2017

\section{References}

1. Wang X, Yu H, Cai Z, et al. Nonketotic hyperglycemia-related epileptic seizures. Epilepsy Behav Case Rep. 2013;1:77-8.

2. Maccario M, Messis CP, Vastola EF. Focal seizures as a manifestation of hyperglycemia without ketoacidosis. A report of seven cases with review of thw literature. Neurology. 1965;15:195-206.

3. Maccario M. Neurological dysfunction associated with nonketotic hyperglycemia. Arch Neurol. 1968;19(5):525-34.

4. Singh BM, Gupta DR, Strobos RJ. Nonketotic hyperglycemia and epilepsia partialis continua. Arch Neurol. 1973;29(3):187-90.

5. Lammouchi T, Zoghlami F, Ben Slamia L, et al. Epileptic seizures in nonketotic hyperglycemia. Neurophysiol Clin. 2004;34(3-4):183-7.

6. Scherer C. Seizures and non-ketotic hyperglycemia. Presse Med. 2005;34(15): 1084-6.

7. Huang CW, Tsai JJ, Ou HY, et al. Diabetic hyperglycemia is associated with the severity of epileptic seizures in adults. Epilepsy Res. 2008;79(1):71-7.

8. Tiamkao S, Pratipanawatr T, Tiamkao S, Nitinavakarn B, et al. Seizures in nonketotic hyperglycaemia. Seizure. 2003;12(6):409-10.

9. Lavin PJ. Hyperglycemic hemianopia: a reversible complication of nonketotic hyperglycemia. Neurology. 2005;65(4):616-9.

10. Brick JF, Gutrecht JA, Ringel RA. Reflex epilepsy and nonketotic hyperglycemia in the elderly: a specific neuroendocrine syndrome. Neurology. 1989;39(3):394-9.

11. Peltola J, Kulmala P, Isojärvi J, et al. Autoantibodies to glutamic acid decarboxylase in patients with therapy-resistant epilepsy. Neurology. 2000; 55(1):46-50.

12. Kim DW, Moon Y, Gee Noh H, et al. Blood-brain barrier disruption is involved in seizure and hemianopsia in nonketotic hyperglycemia. Neurologist. 2011;17(3):164-6.

13. Taieb-Dogui T, Harzallah MS, et al. Acute repetitive giratory seizures as a manifestation of nonketotic hyperglycemia. Neurophysiol Clin. 2002;32(4):254-7.

14. Seo DW, Na DG, Na DL, et al. Subcortical hypointensity in partial status epilepticus associated with nonketotic hyperglycemia. J Neuroimaging. 2003;13(3):259-63.

15. Raghavendra S, Ashalatha R, Thomas SV, Kesavadas C. Focal neuronal loss, reversible subcortical focal T2 hypointensity in seizures with a nonketotic hyperglycemic hyperosmolar state. Neuroradiology. 2007;49(4):299-305.

16. Goto H, Kumagai T, Momozaki N. MRI findings of occipital seizures in nonketotic hyperglycemia. Intern Med. 2011;50(4):367-8.

Submit your next manuscript to BioMed Central and we will help you at every step:

- We accept pre-submission inquiries

- Our selector tool helps you to find the most relevant journal

- We provide round the clock customer support

- Convenient online submission

- Thorough peer review

- Inclusion in PubMed and all major indexing services

- Maximum visibility for your research

Submit your manuscript at www biomedcentral.com/submit
Ciomed Central 